\title{
Association between intraoperative heart rate and postoperative myocardial injury in patients following non-cardiac surgery
}

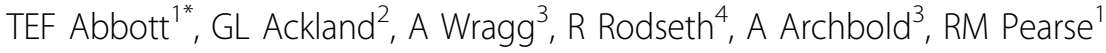 \\ From ESICM LIVES 2015 \\ Berlin, Germany. 3-7 October 2015
}

\section{Introduction}

Approximately $10 \%$ patients who undergo non-cardiac surgery show biochemical evidence of myocardial injury, which is associated with cardiac complications and death [1]. The aetiology and mechanisms of perioperative myocardial injury are incompletely understood. Observational studies have described associations between perioperative tachycardia and postoperative cardiac complications. However, 'tachycardia' was often poorly defined and its association with myocardial injury has not been investigated.

\section{Objectives}

The aim of this study was to determine whether or not intraoperative tachycardia is associated with postoperative myocardial injury.

\section{Methods}

This was a post-hoc analysis of an international observational cohort study of patients undergoing non-cardiac surgery [1]. Maximum and minimum intraoperative heart rates were recorded. Postoperative myocardial injury was defined as serum troponin $\mathrm{T}$ concentration $>0.03 \mathrm{ng} / \mathrm{ml}$ within 30 days of surgery. Heart rate was analysed as a continuous variable and then as a categorical variable divided into five groups $(<45,45-55,56-100$, $101-140,>140 \mathrm{bpm})$. A multivariable logistic regression model was constructed which included known confounding variables to assess for associations between maximum and minimum intraoperative heart rate and myocardial injury. All-cause mortality within 30 days of surgery was a secondary outcome measure.

\begin{tabular}{lcrrr}
\hline \multicolumn{3}{c}{ Mortality } & \multicolumn{2}{c}{ MINS } \\
\hline Heart rate group & odds ratio & p-value & odds ratio & p-value \\
\hline Maximum heart rate & 1.006 & $<0.001$ & 1.002 & 0.027 \\
Minimum heart rate & 0.998 & 0.551 & 0.993 & $<0.001$
\end{tabular}

Multivariable logistic regression models. The mortality analysis was corrected for age, history of peripheral vascular disease, history of COPD, neurosurgery, urgent/emergency surgery, major surgery and the presence of postoperative MINS. The myocardial injury (MINS) analysis was corrected for age, male sex, eGFR, urgent/emergency surgery and history of: atrial fibrillation, diabetes mellitus, hypertension, congestive cardiac failure, coronary artery disease, peripheral vascular disease and stroke or TIA.

Figure 1 Maximum and minimum intraoperative heart rate - multivariable model 


\section{Results}

1,197 patients (7.9\%) of 15,792 patients showed evidence of myocardial injury and 315 (2.0\%) died. Maximum intraoperative heart rate was associated with myocardial injury and mortality. Minimum intraoperative heart rate was negatively associated with myocardial injury, but not mortality (figure 1). Maximum intraoperative heart rate $>140 \mathrm{bpm}$ was more strongly associated with myocardial injury than heart rate 101-140 (Odds ratios 1.67, $\mathrm{p}=0.075 \mathrm{vs}, 1.18, \mathrm{p}=0.057$; reference group heart rate 55-101 bpm).

\section{Conclusion}

Intraoperative tachycardia was associated with adverse postoperative outcomes. Further work is required to refine the relation between perioperative heart rate and post-operative clinical outcomes and whether or not heart rate modulating drugs reduce perioperative myocardial injury.

\section{FUNDING}

TA is supported by an MRC/BJA Clinical Research Training Fellowship.

\section{Authors' details}

${ }^{1}$ Queen Mary University of London, William Harvey Research Institute, London, United Kingdom. ${ }^{2}$ University College London, London, United Kingdom. ${ }^{3}$ Barts Health NHS Trust, London, United Kingdom. ${ }^{4}$ University of KwaZulu-Natal, Durban, South Africa.

Published: 1 October 2015

\section{Reference}

1. Devereaux PJ, Chan MT, et al: Vascular Events In Noncardiac Surgery Patients Cohort Evaluation Study I. JAMA 2012, 307(21):2295-304.

\section{Submit your manuscript to a SpringerOpen ${ }^{\circ}$ journal and benefit from:}

- Convenient online submission

- Rigorous peer review

- Immediate publication on acceptance

- Open access: articles freely available online

- High visibility within the field

- Retaining the copyright to your article 